\title{
Atypical Disseminated Skeletal Tuberculosis Mimicking Metastasis on PET-CT and MRI
}

\author{
Seong Woo Go ${ }^{1}$, Hea Yon $\mathrm{Lee}^{2}$, Chae Hun $\mathrm{Lim}^{3}$, Won-Hee Jee ${ }^{3}$, Young-Pil Wang ${ }^{5}$, \\ Ie Ryung Yoo ${ }^{4}$ and Ji Young Kang ${ }^{2}$
}

\begin{abstract}
Multifocal skeletal tuberculosis is a very rare manifestation of tuberculous infection. The multiple bone lesions of multifocal skeletal tuberculosis are difficult to differentiate from metastasis, even when performing 2-[fluorine-18]-fluoro-2-deoxy-D-glucose (FDG) positron emission tomography (FDG PET/CT) or magnetic resonance imaging (MRI). A 25-year-old man presented with an abnormal chest X-ray. Radiologically, there were extensive osteolytic lesions on the skull, along the entire spine and on the ribs and both iliac bones, suggesting a diagnosis of bone metastasis. On FDG PET/CT, intensely increased F-18 FDG lesions were observed. A bone biopsy with a microbiologic study revealed a tuberculous infection. Follow-up PET/CT performed after treatment showed marked improvement in the extensive FDG uptake lesions.
\end{abstract}

Key words: spinal tuberculosis, osteoarticular, tuberculosis, whole spine MRI, PET/CT scan, metastasis

(Intern Med 51: 2961-2965, 2012)

(DOI: 10.2169/internalmedicine.51.8347)

\section{Introduction}

Skeletal tuberculosis (TB) accounts for approximately 10$20 \%$ of all cases of tuberculosis infection (1). Multifocal skeletal TB, an extremely rare manifestation, is defined as the involvement of two or more bones or joints in a TB infection and accounts for approximately 5\% of all cases of skeletal TB (2). The diagnosis of multifocal skeletal TB is frequently delayed due to its rarity and vague symptoms. Moreover, it is difficult to differentiate multifocal skeletal TB from other multiple bone lesions, including bone metastases, based on clinical or radiologic findings only. In particular, making a diagnosis of multifocal skeletal TB is challenging when MRI findings show unusual manifestations of tuberculous spondylitis. To the best of our knowledge, there are only two reports of magnetic resonance (MR) imaging of tuberculous spondylitis mimicking skeletal metastasis that showed limited areas $(3,4)$. We herein report a case of atypical disseminated skeletal tuberculosis mimicking metastasis that involved the entire spine, skull base, iliac bones, ribs, left scapula and acromion.

\section{Case Report}

A 25-year-old man visited our hospital with incidentally detected abnormalities on chest radiograph performed at a health screening. He had lost approximately $20 \mathrm{~kg}$ in weight during the previous year and complained of vague low back pain. He did not have any significant past medical history, including human immunodeficiency virus (HIV) or hepatitis B virus (HBV) infection. A complete blood count (CBC) showed a leukocyte count of $6,960 / \mathrm{uL}$ with $70.8 \%$ neutrophils and $15.8 \%$ lymphocytes (normal: $20-44 \%$ ) and a decreased hemoglobin level (12.6 g/dL, normal: 13.0-18.0 g/ dL). A blood chemistry analysis showed an Lactate dehydrogenase (LDH) level of $640 \mathrm{U} / \mathrm{L}$ (normal: $250-450 \mathrm{U} / \mathrm{L}$ ) and an albumin level of $3.9 \mathrm{~g} / \mathrm{dL}$ (normal: $3.5-5.5 \mathrm{~g} / \mathrm{dL}$ ).

${ }^{1}$ Department of Internal Medicine, The Catholic University of Korea College of Medicine, Korea, ${ }^{2}$ Division of Pulmonology, Department of Internal Medicine, The Catholic University of Korea College of Medicine, Seoul St. Mary's Hospital, Korea, ${ }^{3}$ Department of Radiology, The Catholic University of Korea College of Medicine, Seoul St. Mary's Hospital, Korea, ${ }^{4}$ Department of Nuclear Medicine, The Catholic University of Korea College of Medicine, Seoul St. Mary's Hospital, Korea and ${ }^{5}$ Department of Thoracic Surgery, The Catholic University of Korea College of Medicine, Seoul St. Mary's Hospital, Korea

Received for publication June 11, 2012; Accepted for publication July 17, 2012

Correspondence to Dr. Ji Young Kang, rkdwldud@catholic.ac.kr 


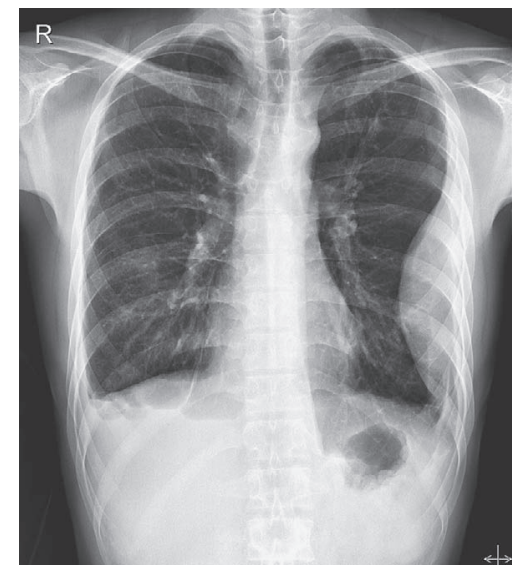

Figure 1. An initial chest posterior-anterior plain radiograph showing loculated pleural effusion or chronic empyema in the lateral portion of the left hemithorax. Bilateral pleural thickening in the costophrenic angles is noted.

A chest radiograph showed what appeared to be either loculated pleural effusion or a pleural-based mass in the left lower thorax (Fig. 1). Computed tomography (CT) showed multifocal, disseminated and purely osteolytic lesions along the spinous and transverse processes of the cervical spine, the vertebral bodies and the ribs. The osteolytic areas also showed minimal soft tissue mass formations. There was no evidence of bone remodeling. A small number of tiny nodules were noted in both upper lobes and linear density was observed in the left upper lobe (Fig. 2). At first, we assumed the pleural manifestations likely indicated a tuberculous infection; however, the disseminated, mottled and pure osteolytic foci replaced with soft tissue lesions in the skeletal system independently indicated metastasis. For further evaluation, a (PET/CT) scan was obtained, which showed disseminated avid F18-(FDG) uptake foci in the skeletal system (maximum Standardized uptake value: 17.4). Additionally, focal physiologic activity was observed in the proximal ascending colon, cecum and terminal ileum that required differentiation from premalignant adenoma (Fig. 3a). The patient underwent a (CT)-guided biopsy of the fifth thoracic spinal body and colonoscopy to rule out primary malignancy. The bone specimen (Fig. 4) showed chronic granulomatous inflammation with caseous necrosis and multinucleated giant cells consistent with mycobacterial infection. Colonoscopy showed irregular ulcerative lesions in the terminal ileum, and the histologic findings showed diffuse necrosis and a granuloma without any evidence of malignancy.

At that time, because we could not completely exclude the possibility of bone metastasis, the patient underwent whole spine MRI after the bone biopsy. On spine MRI, the cervicothoracolumbar spine appeared to have been replaced by multiple masses and focal extraosseous paraspinal masses were observed (Fig. 5). The intervertebral disc space was preserved and neither subligamentous spread nor abscesses were observed in the spine. However, because the imaging findings of MRI and FDG PET/CT were suggestive of bone metastasis from a pleural tumor, we performed a pleural biopsy and bronchoscopy. The pleural biopsy showed chronic inflammation infiltrated with epitheloid cells and a positive TB polymerase chain reaction. During bronchoscopy, no specific endobronchial lesions were observed and bronchial washing/brushing was completed in the right upper lobe and the left lower lobe. Mycobacterial tuberculosis (MTB) was cultured in the bronchial washing fluids two months later. The final diagnosis was disseminated TB involving multiple bones, the pleura, the lungs and the colon. The patient was treated with standard anti-tuberculous chemotherapy, including isoniazid, rifampin, ethambutol and pyrazinamide. Eight months later, follow-up FDG PET/CT showed a marked improvement in the extensive FDG uptake lesions (Fig. 3b).

\section{Discussion}

Skeletal TB primarily develops in the vertebrae, especially in those of the lumbar or thoracic spine. Multifocal skeletal TB most often involves the spine, while tuberculous arthritis involves weight-bearing joints and extraspinal tuberculous osteomyelitis involves the skeletal system (5). Skeletal TB usually occurs in patients with risk factors such as low socioeconomic status and old age or in patients with immunecompromised states such as those with acquired immune deficiency syndrome (AIDS) or those undergoing organ transplantation (6). Skeletal TB primarily results from hematogenous dissemination or lymphogenous spread from primary or reactivated infected foci (6). Concomitant active pulmonary TB occurs in only $30 \%$ of cases of tuberculous osteoarthritis (7). Nonspecific symptoms with slow onset may delay an early and accurate diagnosis of skeletal TB. In the present case, although extensive bone TB with dissemination to multiple organs was observed, the patient initially complained of constitutional symptoms without knowing the onset time and did not have any other immunocompromised conditions such as diabetes, organ transplantation or HIV infection. The incidence of idiopathic (CD) 4+ T- lymphocytopenia is rare; however, some reports have indicated that the disease is associated with severe, disseminated tuberculous infection, such as in the current case $(8,9)$. However, at the time of admission, we did not assess the CD 4+ T lymphocyte count.

The common radiologic changes observed in spinal TB include rarefaction of the vertebral endplates, disc space narrowing, anterior wedging, bony destruction and the development of paraspinal soft tissue abscesses (10). Compared with pyogenic spondylitis, TB spondylitis is primarily found in the anterior elements of the vertebrae with invasion to the paravertabral spaces and preservation of the intervertebral disc spaces. Posterior involvement of the spine is a rare finding with an incidence of approximately $2 \%$ to $10 \%$ worldwide (11). However, our case manifested multiple, disseminated, pure osteolytic lesions without sclerotic changes, while the posterior elements of the spine were diffusely affected without the development of paravertebral soft tissue 
(a)
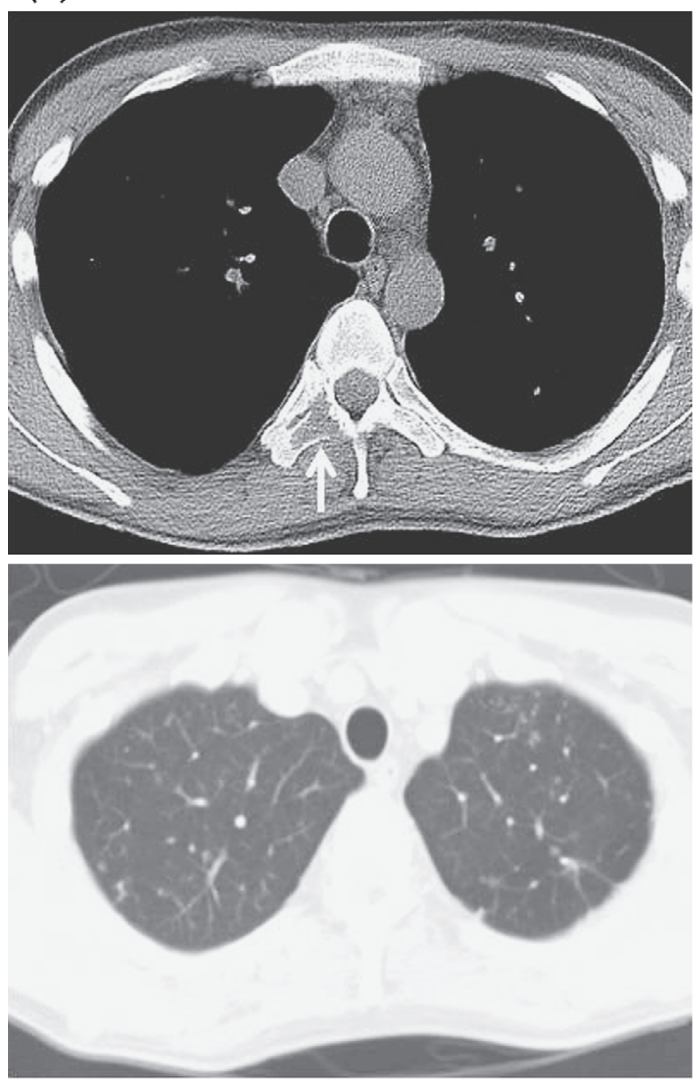

(c) (b)
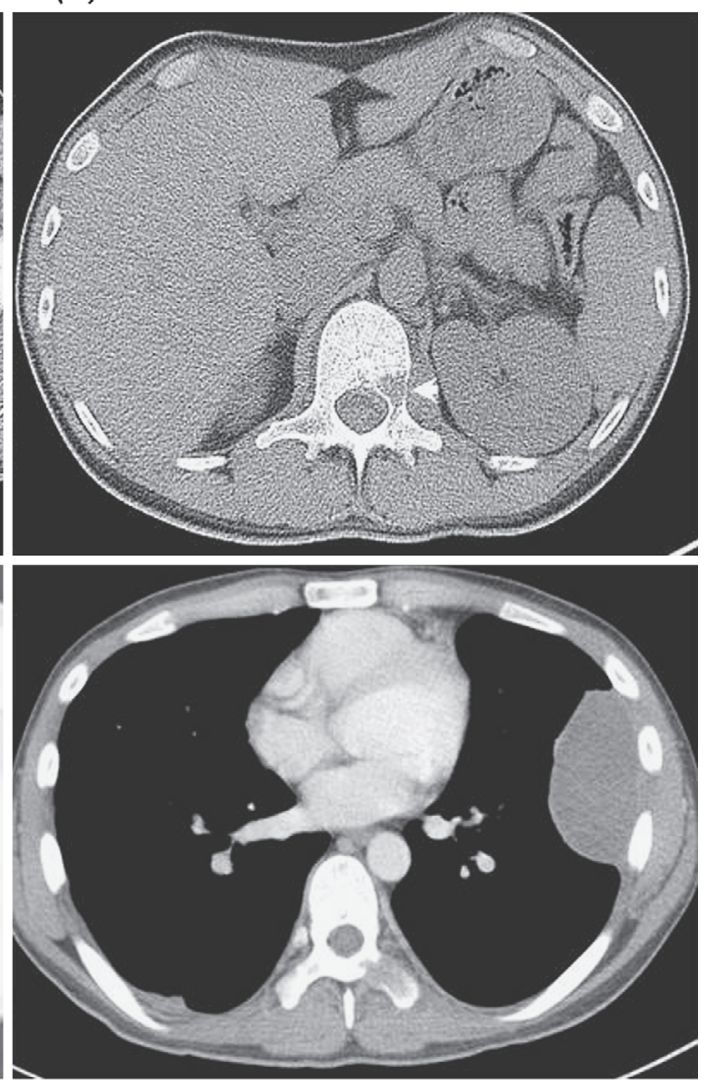

(d)

Figure 2. CT showing multifocal, disseminated and purely osteolytic lesions along the spinous and transverse processes of the spine (arrow and arrowhead). Soft tissue lesions replaced the osteolytic foci. There is no evidence of bone remodeling (a, b). A CT scan with a lung window setting demonstrating a small number of tiny nodules in both upper lobes and linear density in the left upper lobe (c). A large ovoid mass or loculated effusion is noted in the left lateral portion of the pleura (d).

(a)

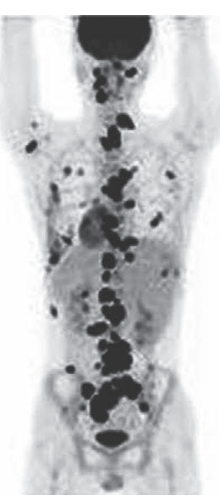

田

Figure 3. A PET/CT scan taken on admission showing disseminated, multiple and avid FDG uptake in the entire body, especially the skeletal system (a). Follow-up scans show a remarkably decreased FDG uptake in all lesions (b).

masses. Regarding MRI of tuberculous spondylitis, the typical findings, as reported by Jung et al. (12), are a welldefined paraspinal abnormal signal, a thin and smooth abscess wall, subligamentous spread to three or more vertebral levels and multiple vertebral or entire body involvement. However, none of these typical (MR) findings were ob-

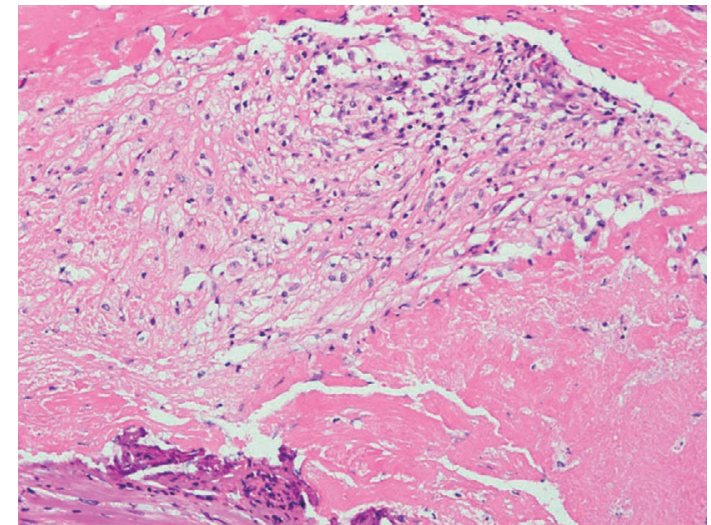

Figure 4. A bone biopsy showing chronic granulomas with caseous necrosis and multinucleated giant cells consistent with mycobacterial infection.

served in the current case except for multiple vertebral body involvement.

Making a definitive diagnosis of multifocal skeletal TB is achieved based on the findings of bone biopsies. Multifocal skeletal TB requires differentiation from eosinophilic granuloma, lymphoma, multiple myeloma and metastasis. Hong et 


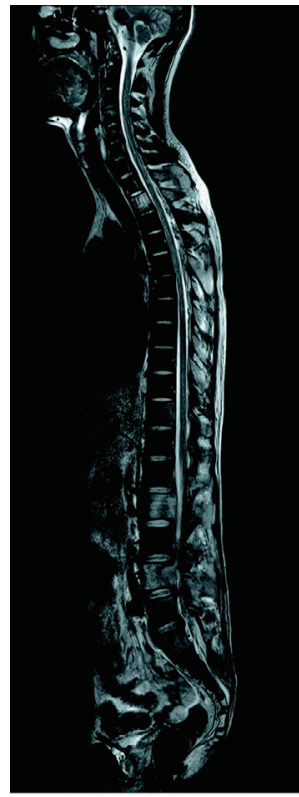

(a)

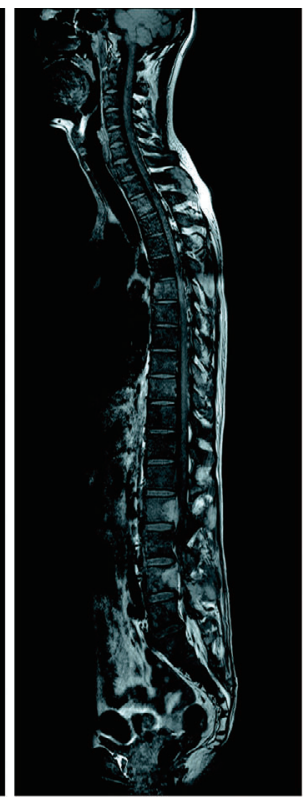

(b)

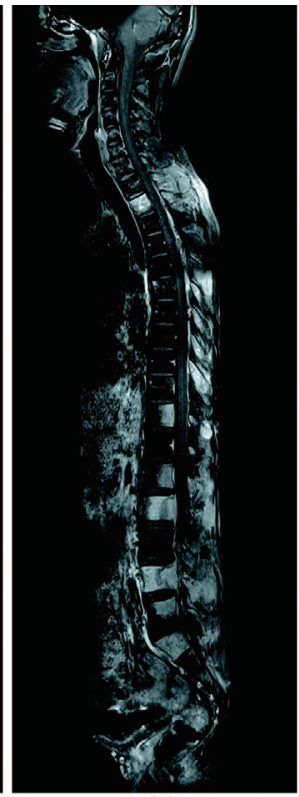

(c)

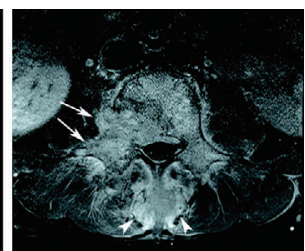

(d)

Figure 5. Whole spinal MR imaging showing diffusely decreased bone marrow signals on T1- and T2- weighted images due to anemia $(\mathbf{a}, \mathbf{b})$. The cervicothoracolumbar spine is replaced by multiple masses, and surrounding marrow edema is observed on the fat-saturated sagittal T2-weighted images (a). On the gadolinium-enhanced T1-weighted images, the enhanced soft tissues masses and marrow edema are more highly accentuated (c). Focal extraosseous paraspinal masses are noted on an axial enhanced T1-weighted image (d, arrow and arrowheads). The interverteral disc space is preserved, and neither subligamentous spread nor definite abscess pockets are observed along the entire spine.

al. reviewed 13 cases of multifocal skeletal TB and reported that most of the patients had four to six lesions and the flat bones were the most commonly involved site (1). He also described a case of 26 tuberculous bone lesions in an immunocompromised patient. Our patient was immunocompetent and had at least 25 tuberculous bone lesions in eight locations: the skull, left scapula, acromion, both ribs, C-T-L spine, both iliac joints, sacrum and other organs such as the pleura, lungs and colon. We performed FDG PET/CT for further evaluation, which showed F-18 FDG uptake lesions in the colon, pleura and lung parenchyma in addition to the bones. It is well known that it is difficult to distinguish metastatic bone lesions from acute inflammatory changes caused by TB using FDG PET/CT because, not only tumors, but also infections and inflammations show increased F-18 FDG uptake. However, FDG PET/CT is helpful for detecting asymptomatic and abnormal lesions and monitoring disease activity after treatment by evaluating decreased F-18 FDG uptake during follow-up, as in the current case. Indeed, Kim et al. (13) showed that F-18 FDG PET/CT is useful for discriminating between residual and nonresidual lesions that occur after treatment in patients with spinal infections, including TB spondylitis.

The treatment of multifocal skeletal tuberculosis involves the administration of four major drugs in a standard antituberculosis regimen over 12 to 18 months. Surgical intervention may be required in cases of severe joint deformity, cold abscesses or necrotic debris. Adjunctive management such as immunotherapy with interferon and thymic peptides may be helpful (14). The prognosis is favorable, and patients with multifocal skeletal TB can be completely cured if the disease is detected early. In a literature review of 17 cases of multifocal skeletal TB occurring between 2000 and 2007, most patients were cured with standard antituberculous medication administered for 12 to 18 months. Among these patients, only two underwent surgery for pus drainage and removal of necrotic tissue, and their outcomes after surgery were good and without the development of any sequelae. Our patient showed marked improvement in the extensive lesions in the pleura, colon and skeletal system after receiving standard anti-tuberculosis medication for eight months. He has continued to take the medication for one year, namely up until the present time. In conclusion, we herein reported an unusual case of disseminated tuberculous infection that demonstrated quite atypical radiologic findings in the skeletal system, thus resulting in confusion with a diagnosis of bone metastasis.

The authors state that they have no Conflict of Interest (COI).

\section{References}

1. Hong L, Wu JG, Ding JG, et al. Multifocal skeletal tuberculosis: experience in diagnosis and treatment. Med Mal Infect 40: 6-11, 2010.

2. Chang DS, Raffi M, McGuinness G, Jagirdar JS. Primary multifo- 
cal tuberculous osteomyelitis with involvement of the ribs. Skeletal Radiol 27: 641-645, 1998.

3. Khattry A, Thulkar S, Das A, Khan SA, Bakhshi S. Spinal tuberculosis mimicking malignancy: Atypical imaging features. Indian Journal of Pediatrics 74: 297-298, 2007, DOI: 10.1007/s12098007-0049-3

4. Jahng J, Kim YH, Lee KS. Tuberculosis of the lower lumbar spine with an atypical radiological presentation - a case mimicking a malignancy. Asian Spine J 1: 102-105, 2007, DOI: 10.4184/ asj.2007.1.2.102

5. Moore SL, Rafii M. Imaging of musculoskeletal and spinal tuberculosis. Radiol Clin North Am 39: 329-342, 2001.

6. De Backer AI, Mortelé KJ, Vanhoenacker FM, Parizel PM. Imaging of extraspinal musculoskeletal tuberculosis. Eur J Radiol 57: 119-130, 2006.

7. Chapman M, Murray RO, Stoker DJ. Tuberculosis of the bone and joints. Semin Roentgenol 14: 266-282, 1979.

8. Kony SJ, Hane AA, Larouze B, et al. Tuberculosis-associated severe CD4+ T-lymphocytopenia in HIV-seronegative patients from Dakar. SIDAK Research Group. J Infect 41: 167-171, 2000.
9. Pilheu JA, De Salvo MC, Gonzalez J, et al. CD4+ Tlymphocytopenia in severe pulmonary tuberculosis without evidence of human immunodeficiency virus infection. Int $\mathbf{J}$ Tuberc Lung Dis 1: 422-426, 1997.

10. De Vuyst D, Vanhoenacker F, Gielen J, Bernaerts A, De Schepper AM. Imaging features of musculoskeletal tuberculosis. Eur Radiol 13: 1809-1819, 2003.

11. Abdelwahab IF, Camins MB, Hermann G, Klein MJ. Vertebral arch or posterior spinal tuberculosis. Skeletal Radiol 26: 737-740, 1997.

12. Jung NY, Jee WH, Ha KY, Park CK, Byun JY. Discrimination of tuberculous spondylitis from pyogenic spondylitis on MRI. Am J Roentgenol 182: 1405-1410, 2004.

13. Kim SJ, Kim IJ, Suh KT, Kim YK, Lee JS. Prediction of residual disease of spine infection using F-18 FDG PET/CT. Spine (Phila Pa 1976) 34: 2424-2430, 2009.

14. Achkar JM, Casadevall A, Glatman-Freedman A. Immunological options for the treatment of tuberculosis: evaluation of novel therapeutic approaches. Expert Rev Anti Infect Ther 5: 461-474, 2007.

(C) 2012 The Japanese Society of Internal Medicine http://www.naika.or.jp/imonline/index.html 